The University of San Francisco

USF Scholarship: a digital repository @ Gleeson Library |

Geschke Center

2014

\title{
Resonant-Frequency Discharge in a Multi-Cell Radio Frequency Cavity
}

S. Popović

J. Upadhyay

J. Mammosser

Milka Nikolic

University of San Francisco, mnikolic@usfca.edu

L. Vušković

Follow this and additional works at: http://repository.usfca.edu/phys

Part of the Physics Commons

\section{Recommended Citation}

Popović, S., Upadhyay, J., Mammosser, J., Nikolić, M., Vušković, L. Resonant-frequency discharge in a multi-cell radio frequency cavity (2014) Journal of Applied Physics, 116 (17), art. no. 173301. http://dx.doi.org/10.1063/1.4900994

This Article is brought to you for free and open access by the College of Arts and Sciences at USF Scholarship: a digital repository @ Gleeson Library | Geschke Center. It has been accepted for inclusion in Physics and Astronomy by an authorized administrator of USF Scholarship: a digital repository @ Gleeson Library | Geschke Center. For more information, please contact repository@usfca.edu. 


\section{Resonant-frequency discharge in a multi-cell radio frequency cavity}

S. Popović, J. Upadhyay, J. Mammosser, M. Nikolić, and L. Vušković

Citation: Journal of Applied Physics 116, 173301 (2014); doi: 10.1063/1.4900994

View online: http://dx.doi.org/10.1063/1.4900994

View Table of Contents: http://aip.scitation.org/toc/jap/116/17

Published by the American Institute of Physics

Applied Physics Reviews 


\title{
Resonant-frequency discharge in a multi-cell radio frequency cavity
}

\author{
S. Popović, ${ }^{1}$ J. Upadhyay, ${ }^{1}$ J. Mammosser, ${ }^{2}$ M. Nikolić, ${ }^{1}$ and L. Vušković ${ }^{1}$ \\ ${ }^{1}$ Department of Physics, Center for Accelerator Sciences, Old Dominion University, Norfolk, Virginia 23529, \\ USA \\ ${ }^{2}$ Thomas Jefferson National Accelerator Facility, Newport News, Virginia 23606, USA
}

(Received 28 July 2014; accepted 22 October 2014; published online 5 November 2014)

\begin{abstract}
We are reporting experimental results on a microwave discharge operating at resonant frequency in a multi-cell radio frequency (RF) accelerator cavity. Although the discharge operated at room temperature, the setup was constructed so that it could be used for plasma generation and processing in fully assembled active superconducting radio-frequency cryo-module. This discharge offers a mechanism for removal of a variety of contaminants, organic or oxide layers, and residual particulates from the interior surface of RF cavities through the interaction of plasma-generated radicals with the cavity walls. We describe resonant RF breakdown conditions and address the issues related to resonant detuning due to sustained multi-cell cavity plasma. We have determined breakdown conditions in the cavity, which was acting as a plasma vessel with distorted cylindrical geometry. We discuss the spectroscopic data taken during plasma removal of contaminants and use them to evaluate plasma parameters, characterize the process, and estimate the volatile contaminant product removal. C 2014 AIP Publishing LLC. [http://dx.doi.org/10.1063/1.4900994]
\end{abstract}

\section{INTRODUCTION}

Study of the interaction of solid surface with environment (vacuum, gas, or plasma) becomes prohibitively complex when the surface is contaminated. These problems are augmented in large vessels, such as accelerator cavities or fusion plasma reactors. The necessity of cleaning and degassing of large vacuum vessels was first acknowledged by the thermonuclear research community in late seventies. $^{1,2}$ Research effort was concentrated at conditioning Tokamak walls with a d.c. glow discharge in hydrogen, in order to reduce the effective nuclear charge, $\mathrm{Z}_{\text {eff }}$, close to one. Decontamination studies of Tokamak walls have been renewed in 1990s. Vigorous activity on glow discharge, electron cyclotron resonance discharge, and ion cyclotron resonance discharge cleaning was reported. ${ }^{3}$ In Tokamak Experiment for Technology Oriented Research (TEXTOR), oxygen gas flow was applied to hot walls to remove codeposits containing deuterium. TEXTOR did not experience any long term adverse consequences after the use of oxygen to remove deuterium, and high performance plasma operation was recovered shortly after the treatment. ${ }^{3}$

In-vessel components of International Thermonuclear Experimental Reactor (ITER) ${ }^{4,5}$ are subject to plasmainduced erosion and subsequent deposition of eroded material, which has to be removed during maintenance. An out-of-vessel technique was proposed to clean the elements, whereby a contaminated component is dismounted from the vessel and placed in an auxiliary cleaning reactor, where a d.c. glow discharge was applied. ${ }^{4}$ Ongoing research has been active on treatment of walls of a plasma reactor. ${ }^{6}$

Dylla ${ }^{7}$ has acknowledged the beneficial role of glow discharge conditioning as in situ procedure and proposed this application to magnetic fusion devices and to particle accelerator systems. This idea proved to be difficult to implement because it was required to operate at cryogenic condition in the vessels with non-cylindrical geometry. The work on gas discharge conditioning has restarted with the primary idea to excite a radio-frequency discharge in an accelerator cavity at resonant conditions, applying a traveling wave, in the manner how the accelerator itself operates. It has been shown ${ }^{8}$ that plasma cleaning could reduce field emission, clean radio frequency (RF) surfaces leading to stable vacuum in the cavity and reduce the number of accelerator interlock trips. Additionally, this method of cleaning should remove lossy, conductive oxides from the surface thus reducing the RF losses to the cryogenic bath. Moreover, the ability of plasma to follow the shape of the hollow structure will make it most suitable for cleaning complex, non-symmetric surfaces in geometrically complicated RF cavities such as quarter wave or spoke designs. ${ }^{9}$ Although by far smaller than the fusion reactor vessel, the elliptical RF cavity still falls in the category of relatively large industrial containers, with volume sometimes exceeding $10^{5} \mathrm{~cm}^{3}$. The first issue is, therefore, related to creating conditions for stable discharge over the whole vessel's volume. Second, the discharge has to be generated without disturbing the cavity set-up, either upon removal of the cavity from the accelerator, or by running the discharge, when the cavity is integrated in the accelerator system. Third, the contaminants have to be produced in a volatile form, and evacuated from the vessel without redeposition. Accelerator cavity's geometry is defined by the resonant nature of its application, and it is natural to rely on the resonance conditions to generate a discharge within the cavity.

Phenomenology of the resonant microwave discharge can be divided in two subject areas, microwave breakdown ${ }^{10,11}$ at resonant conditions and sustained plasma operation. $^{12,13}$ The resonance condition can be shifted by up to $100 \mathrm{MHz}$ (Refs. 14 and 15) and the frequency of the power source would have to be adjusted. In present work, we are using a multi-cell cavity and the resonance is manifested in 
several modes. It is natural to choose the $\pi$ mode since one wants uniform penetration of produced radicals to every contaminated pocket in the area.

This paper continues with Sec. II where we discuss the mechanism of resonant microwave breakdown in the cavity with a distorted cylindrical geometry. Although there is a variety of a possible breakdown marker, we have restricted ourselves on the onset of optical emission from the cavity and verified the inception by the drop in the transmitted power through the cavity. In Sec. III, we describe the experimental set-up and its consequences to resonant breakdown conditions and to plasma diagnostics. In Sec. IV, we present the actual results on resonant microwave breakdown, discuss the parameter range where the plasma is sustained and we present preliminary data on volatile impurity spectra, outline the optimal pressure range, and estimate the time scale of plasma cleaning with $\mathrm{Ar} / \mathrm{O}_{2}$ mixture. We conclude with confirmation of the versatility and effectiveness of the technique.

\section{MICROWAVE BREAKDOWN IN RESONANT CAVITIES}

In an experiment of this type, we encounter three different resonant phenomena. First, the cavity at hand is the accelerating structure, designed to provide the energy transport along its axis with minimal losses. It is composed of 5 cells designed as identical individual resonators. Hence its driving frequency resonance has a complex band structure with five peaks, corresponding to five modes. In this case, the $\pi$-mode was used, and its resonance was attained by precise adjustment of generator frequency. The driving frequency resonance is involved in the resonant microwave breakdown, which is discussed in this section and in Sec. IV A. There is no plasma in the cavity prior to breakdown, and the driving frequency resonance is the single resonant phenomenon involved in the power coupling.

The second resonance in this experiment corresponds to the condition that plasma frequency is equal to the resonant driving frequency of the cavity $\pi$-mode. This condition is fulfilled by raising the coupling power while keeping the driving frequency of the cavity constant. With rising coupling power, electron density is increased and, consequently, the reflectivity of the plasma column is increased. When the electron density in the plasma reaches the critical value, the transmitted power through the plasma drops to a low value. At low pressure, when the electron collision frequency is negligible in comparison to the resonant frequency, the propagation vector of the electromagnetic field drops to zero. ${ }^{10}$ This effect is indicative for the second, plasma resonance, when plasma frequency is equal to the resonant driving frequency. This condition is used in the Sec. IV B to determine the electron density in the plasma column.

Once the plasma is generated in the cavity, the resonant driving frequency is shifted due to the losses in the plasma. The third resonance is then attained by adjusting the frequency of the coupling power to obtain the glow of maximum intensity. The shifts of the resonant maxima due to losses induced by the plasma column affect the driving frequency range. They were also measured and are discussed in
Sec. IV B in order to determine the needed frequency tuning range of the microwave generator.

We proceed with the analysis of the resonant microwave breakdown, where the first resonance is involved. When the electron-impact ionization presents a dominant electron gain process and the diffusion loss and the attachment are the electron loss mechanisms, the threshold electric field $E_{t}$ in the diffusion-dominated branch of the microwave breakdown scheme $^{10}$ is given by

$$
\nu_{i}\left(E_{t}\right)+\nu_{a}=\frac{D(p, I P)}{\Lambda^{2}},
$$

where $\nu_{i}$ and $\nu_{a}$ are the ionization and attachment collision rates, respectively, $D(p, I P)$ is the electron diffusion coefficient as a function of $p$ (the gas pressure) and $I P$ (the ionization potential), and $\Lambda$ is the effective diffusion length. In the pressure range of our interest, from $p=0.01$ Torr to $p=0.03$ Torr, the attachment rate on the Oxygen atoms is negligible with respect to the diffusion losses, which allows us to reduce the microwave breakdown to the only two remaining processes, ionization gain and diffusion loss.

Assuming that all power at breakdown goes to ionization, ${ }^{10}$ we obtain

$$
\nu_{i}=\frac{e E_{t}^{2}}{m \cdot I P \cdot \nu_{m}}\left(\frac{\nu_{m}^{2}}{\nu_{m}^{2}+\omega^{2}}\right),
$$

where $m$ and $e$ are the mass and charge of an electron, respectively, $\nu_{m}$ is the electron-neutral collision frequency, and $\omega$ is the microwave frequency for the $\pi$-mode resonance. For $\omega^{2} \gg \nu_{m}^{2}$ Eq. (2) simplifies to

$$
\nu_{i} \cong \frac{e E^{2} \nu_{m}}{m \cdot I P \cdot \omega^{2}} .
$$

Therefore, a simplified breakdown criterion is valid

$$
\frac{e E_{t}^{2} \nu_{m}}{m \cdot I P \cdot \omega^{2}}=\frac{D(p, I P)}{\Lambda^{2}} .
$$

The cavity has cylindrical symmetry, where the effective diffusion length is given by

$$
\frac{1}{\Lambda^{2}}=\frac{1}{L} \int_{0}^{L}\left[\left(\frac{\pi}{L}\right)^{2}+\left(\frac{2.405}{R(z)}\right)^{2}\right] d z
$$

where $R(z)$ is the cavity profile and $L$ is the length of a cavity cell, usually taken as $L \cong c / 2 \nu_{r} \cong 10 \mathrm{~cm}$ for the resonant frequency $\nu_{r} \approx 1.5 \mathrm{GHz}$.

Applying the usual relation ${ }^{11}$ for transverse electron diffusion coefficient in the $\mathrm{Ar} / \mathrm{O}_{2}$ mixture (90:10) and the mass action rules,

$$
\begin{aligned}
\ell & =0.9 \ell_{A r}+0.1 \ell_{O_{2}}, \\
\nu_{m} & =0.9 \nu_{m, A r}+0.1 \nu_{m, O_{2}}, \\
I P & =0.9 I P_{A r}+0.1 I P_{O_{2}},
\end{aligned}
$$

where $\ell$ is the effective electron mean free path. The full quantitative formulation for the microwave breakdown in 
cylindrical geometry can be made at low pressures $(\mathrm{p}<0.1$ Torr), where one can use the simple formula for the diffusion coefficient, ${ }^{11}$

$$
D=\frac{\ell^{2} \nu_{m}}{3}
$$

In addition, this simple breakdown model is based on the assumption that (a) the attachment collision rate is negligible in comparison to the ionization rate and (b) the diffusion coefficient is constant over the cavity volume. It is shown in Sec. IV that this approximate model describes rather well the breakdown conditions at the cavity axis. Note that the breakdown condition is given in the form of an implicit relationship between the breakdown electric field and pressure, but the raw breakdown data are obtained as the relation between the measured power that was transmitted into the cavity volume and average pressure in the cavity. In Sec. III, we define the relation between the power and electric field, so that the breakdown condition could be adequately tested.

\section{EXPERIMENTAL SET UP AND PLASMA DIAGNOSTICS}

In this paper, we address the ex situ discharge process at room temperature and in the pressure range below 0.1 Torr predominantly in $\mathrm{Ar}$ mixed with a small quantity of $\mathrm{O}_{2}$ (about $10 \%$ or less). In our earlier and ongoing work, we have successfully used a more reactive gas mixture with a substantial amount of Chlorine diluted in $\operatorname{Argon}^{16,17}$ to obtain robust etching rates of $\mathrm{Nb}$ samples for a superconductive RF cavity. In this work, we use a five-cell $1.497 \mathrm{GHz}$ (Continuous Electron Beam Accelerator Facility) CEBAF cavity at resonant power coupling conditions, the same as in the actual set-up inside the accelerator cryomodule. The preliminary work was reported at a conference. ${ }^{18}$ This cavity exhibits an exemplary departure from a pure cylindrical geometry, which had a consequence in a partial filling of the cavity with plasma. The cavity has five modes with resonances densely located around $1.5 \mathrm{GHz}$. Field distributions in the full-cell mode ( $\pi$ mode) and one part-cell mode $(4 \pi / 5$ mode) are given in Fig. 1. Intense field regions illustrate the expected plasma layout after breakdown in one of the modes. The $\pi$ mode is expected to excite uniformly the resonant discharge in all five cells, but the $4 \pi / 5$ is likely to excite two plasma columns, avoiding the excitation of the central cell.

A five-cell cavity was taken randomly from the Thomas Jefferson National Accelerator Facility (TJNAF) stock and coupled into the vacuum system and the microwave circuit as shown in Fig. 2.

To provide the best possible power coupling at room temperature, a mechanical squeezing tuner was used on the short-circuit terminator side of the FPC. Although the tuner helped to determine an optimal standing wave pattern for cross-coupling the power into the cavity, this type tuner cannot be used at cryogenic conditions. At those conditions, an external waveguide stub tuner would have to be used to change the coupling instead of the FPC squeezing tuner. The power was coupled to the cavity through a circular aperture

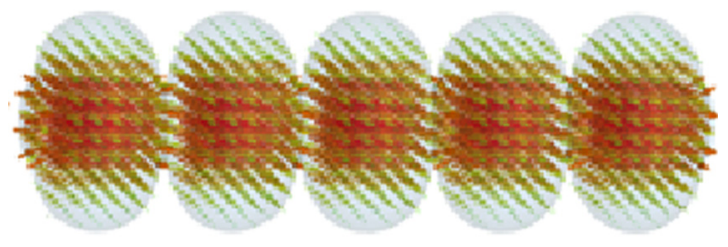

(a)

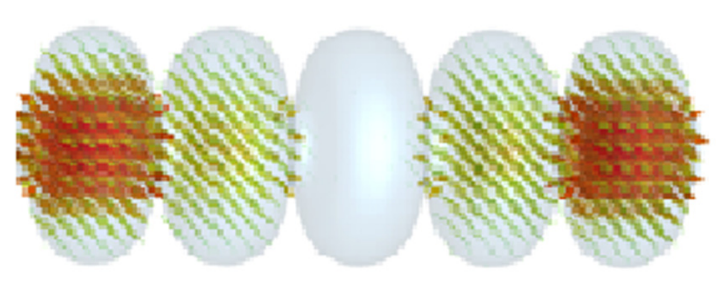

(b)

FIG. 1. Electric field distribution in the five cell accelerator cavity: (a) $\pi-$ mode and (b) $4 \pi / 5-$ mode.

of the same diameter as the cavity iris that was $70 \mathrm{~mm}$. Center of the aperture was located at the maximum of the standing wave pattern in the feeding waveguide system with the mechanical tuner applied. Power was supplied by the tunable microwave generator (Microwave Amps, Ltd., Model AM87-1-4 S-56-53 R) with the tuning covering full resonant frequency range accounting for all five cavity modes and detuning effects due to plasma processes. Signal generator for the microwave amplifier was Wavetek Make (2-2200) MHz range. Power was transmitted through coaxial cable to the bidirectional coupler, which was connected to the L-band waveguide system. The E sides of the waveguide were tapered to fit the dimensions of the actual microwave circuit elements in the cryomodule, including the ceramic window, transparent to the microwave transmission. In this experiment, the window separated the vacuum section from the power feeding section of the microwave system.

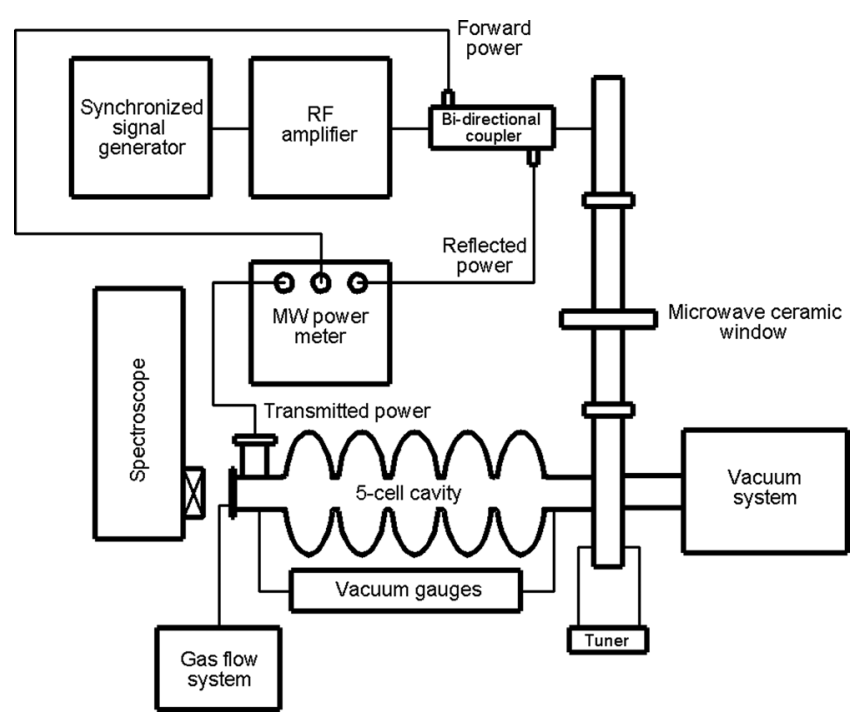

FIG. 2. Scheme of the experimental set-up for the five cell cavity resonant microwave plasma generation. 
At one end, the cavity is terminated with a vacuum flange. The flange contained a small-aperture window for plasma visualization and end-on spectroscopy. The port on a higher order mode coupler is used for the transmitted microwave power probe. Vacuum is achieved with a compact Agilent system, Model TV301. Gas mixture is applied through a port located on the opposite side of the cavity. Plasma was generated in a five-cell cavity where a pressure gradient was maintained between the gas manifold at the upstream and pumping orifice at the downstream side of the cavity. In the operating pressure range, the ratio of the pressure at the upstream and downstream end of the cavity was about three due to the relatively large length of the cavity. We note that the pressure gradient varies between 0.01 and $0.1 \mathrm{Torr} / \mathrm{m}$. Therefore, there is a substantial variation of the reduced electric field in the cavity not only due to the electric field distribution but also due to the density variation. This variation can be as large as a factor of five along the cavity axis. As a consequence, the exact location of breakdown was unclear, but the plasma column expanded axially faster than the time-averaging diagnostic equipment could record.

A set of temperature sensors was distributed over the external wall of the cavity to measure temperature distribution along the cavity. Variations in temperature were not substantial.

Power measurements were carried on using three RF power meters, one each located on the forward and reflected-power side of the bidirectional coupler, and the third on the field sampling probe, a small electric field antenna, which samples the field in the cavity cells. This field sampling probe is standard hardware on all cryomodules. Each power meter is calibrated to a reference, RF power, and to the frequency of the device to be measured in this case the cavity. Calibration of the RF paths is performed by sending a reference RF signal in the same frequency range through the directional coupler and transmitted power cables, and comparing the reference power and the power measured on each of the three power meters. In this way, a correction factor can be calculated for each of the signal paths.

To convert power measurements into the values of the effective electrical field at the cavity axis, the following procedure was used. First, we evaluated cavity power and axial field from the sensor data. Incident (forward), reflected power and transmitted power are given by

$$
\begin{aligned}
P_{i n} & =C_{i n} \times C C F \times P_{i n m}, \\
P_{r e f} & =C_{r e f} \times C C F \times P_{r e f m}, \\
P_{t} & =C_{t r} \times P_{t r m},
\end{aligned}
$$

where $P_{i n m}, P_{r e f m}$, and $\mathrm{P}_{\text {trm }}$ are the measured signals from power probes; $C_{i n}, C_{r e f}$, and $C_{t r}$ are the power correction factors; and $C C F$ is the cable conversion factor. Dissipated power into the cavity volume was

$$
P_{\text {diss }}=P_{\text {in }}-P_{r e f}-P_{t r} .
$$

When the plasma was on, transmitted power $\left(P_{t r}\right)$ was negligible.
Niobium cavity $Q$-factor at room temperature was low, $Q=9000$. There were five segments in the cavity. The cavity impedance was therefore 864000 for $R / Q=96 \Omega$.

Voltage at the cavity was

$$
V=\sqrt{2 P_{\text {diss }} R}=930 \sqrt{P_{\text {diss }}} .
$$

Electric field to be associated with the plasma for the purpose of breakdown analysis is given by

$$
E=\frac{V}{L}=\frac{2 V}{\lambda} \cong 93 \sqrt{P_{\text {diss }}} \text {. }
$$

\section{RESULTS AND DISCUSSION}

\section{A. Resonant frequency breakdown}

Plasma was successfully generated at a number of points in the pressure range 10-30 mTorr in $\mathrm{Ar} / \mathrm{O}_{2}$ mixture (90/10) at the frequency corresponding to the $\pi$-mode, about $1.5 \mathrm{GHz}$. In some occasions, a small amount of $\mathrm{N}_{2}$ was present. The results on microwave breakdown are given in Fig. 3. The breakdown data were taken in the usual way. Power source frequency was set to resonance corresponding to the $\pi$-mode maximum. Pressure in the cavity was set and kept constant, while the generator power was gradually increased until a glow was observed visually. In coincidence, the transmitted power signal at the opposite end of the cavity dropped by several orders of magnitude. Those two coincidental events marked the $\pi$ mode resonant frequency breakdown. Electric field values were then evaluated from the power data gathered just before the breakdown. The error bars combine the statistical uncertainty in power signal readings, and the uncertainties involved in the procedure for the evaluation of the electric field. Pressure values shown in Fig. 3 are the average along the cavity, obtained by averaging of the upstream and downstream pressure readings. The error bars here also reflect the uncertainty in the pressure reading and in the evaluation method used. Referring to Eqs. (4), (5), and (10), the pressure, forward, reflected, and

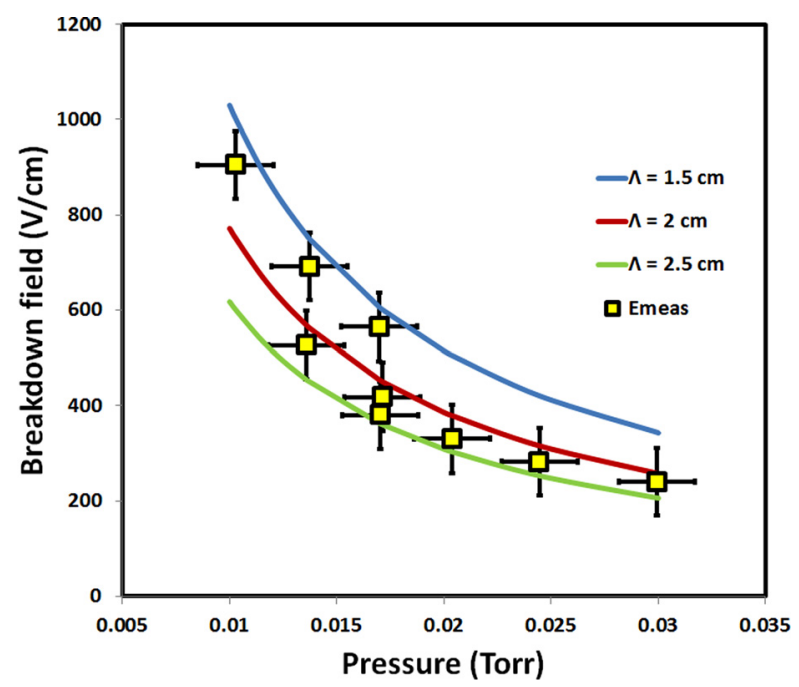

FIG. 3. Microwave breakdown field in the five-cell CEBAF cavity $\pi$ mode at $1.5 \mathrm{GHz}$. 
dissipated power, together with the geometrical parameters were measured directly. Pressure error bars reflect the uncertainty in the flow gradient, which was about $\pm 8 \%$. Power measurements were made with the uncertainty of $\pm 5 \%$ each. Therefore, the uncertainty in the electric field was about $\pm 7.5 \%$, according to Eq. (10) and it was included in Fig. 3.

Solid curves are obtained by applying the approximate breakdown criterion defined in the Section II, for three different effective diffusion lengths, $\Lambda=1.5,2$, and $2.5 \mathrm{~cm}$. The effective diffusion length calculated based on the cavity envelope $R(z)$ given in Eq. (5) is $\Lambda=1.96 \mathrm{~cm} \approx 2 \mathrm{~cm}$. By comparison, the diffusion length for a cylinder of radius $R=3.5 \mathrm{~cm}$, which is the cavity radius at the iris, is $\Lambda \approx 2.4 \mathrm{~cm}$. At the opposite extreme, the diffusion length for a cylinder of radius $R \approx 8.8 \mathrm{~cm}$, which is the cavity radius at equator, is $\Lambda \approx 1.15 \mathrm{~cm}$. We note that the experimental values shown in Fig. 3 do fall in the range $1.5<\Lambda<2.5 \mathrm{~cm}$, but are all concentrated around $\Lambda=2 \mathrm{~cm}$, within the error bars, which justifies the diffusion breakdown model based on the cavity envelope. Therefore, we can discuss the breakdown in the five-cell cavity in the terms of diffusion-dominated regime and use the diffusion as the dominant charged-particle loss in present geometry.

\section{B. Plasma parameters}

Experimental data show that the integral transmission coefficient at discharge inception has dropped to almost zero,

$$
T=\frac{P_{t r}}{P_{\text {in }}}=0.025 \approx 0 .
$$

This is the indication for the critical plasma resonant condition, where the real part of the electromagnetic wave number is approximately zero. This condition can be used for global diagnostics of electron density. Assuming that the collision frequency is low in comparison with the cavity resonant frequency (see Eq. (3)), the critical plasma resonant condition is reduced to ${ }^{10}$

$$
\omega_{p}=\sqrt{\frac{n_{e} e^{2}}{m_{e} \varepsilon_{0}}}=2 \pi f_{r}
$$

where $\omega_{p}$ is the plasma frequency, $n_{e}$ is the electron density, $e$ and $m_{e}$ are the electron charge and mass, respectively, $\varepsilon_{0}$ is the vacuum permeability, and $f_{r}$ is the resonant frequency of the cavity.

Note that this criterion should not be misinterpreted as the cavity resonance. While the cavity resonance is the condition of maximum power coupling to the cavity, the plasma resonance is the condition for maximum reflection of the electromagnetic wave from the plasma column. It represents the condition where the plasma frequency and the coupling power frequency are equal,

$$
f_{r}=\frac{1}{2 \pi} \frac{e}{\sqrt{m_{e} \varepsilon_{0}}} \sqrt{n_{e}} .
$$

This condition leads to the relation between the power resonant frequency and an average electron density along the plasma column in the cavity, ${ }^{10}$

$$
f_{r}(H z) \cong 8980 \sqrt{n_{e}\left(\mathrm{~cm}^{-3}\right)} .
$$

Using the above plasma resonant condition, we arrive at the post-breakdown, sustained plasma density of $n_{e} \approx 2.8 \times 10^{10} \mathrm{~cm}^{-3}$.

After breakdown, we have further observed the glow visually, while we varied the frequency in a limited frequency range with the goal to obtain the new resonance involving the sustained plasma. It turned out that the resonance shift in the presence of the discharge in the cavity varied from mode to mode. For the $4 \pi / 5$ cavity mode, the glow intensity has increased up to $\Delta f \approx 2 \mathrm{MHz}$ above the resonance frequency in the absence of the plasma at which point we stopped increasing the frequency due to window flashover discharge. Therefore, we concluded that the plasmaaffected detuning of the cavity was at least $2 \mathrm{MHz}$. This detuning effect due to the presence of plasma inside the cavity that increases the power loss and causes its resonant frequency to shift was still substantially small compared with the frequency shift results in some other experimental and theoretical work ${ }^{14,15,19,20}$ performed for cylindrical cavities. In those References, the resonance shifts as large as $100 \mathrm{MHz}$ were measured and calculated ${ }^{12}$ for a variety of TE and TM modes, although the standing wave pattern had been still sustained in the plasma, which behaves as a poor conductor with corresponding losses. We note here that in the present experiment, the critical resonant conditions for plasma column have not been attained and the frequency shift may be an order of magnitude larger. Although the frequency shift may have an important diagnostic purpose in the future and the operation on the retuned cavity would produce more effective in situ cleaning due to the so-called "frequency tuning effect," 11 we have concentrated on the effects that plasma had upon the cavity surface and on the spectroscopic determination of plasma parameters. Calculation of the frequency shift induced by the plasma and the validation of the frequency tuning effect by experiment will be of importance for further development of both in situ and ex situ plasma cleaning techniques.

Electron temperature and density were separately evaluated using optical emission spectra, by comparison of neutral Argon and Argon ion (4p-4s) line intensities located in the same wavelength frame and recorded simultaneously. This procedure is described below.

\section{Optical emission spectroscopy (OES)}

Optical spectra from the plasma were taken along the cavity axis using a $0.75 \mathrm{~m}$ Acton, Princeton Instruments monochromator with a $3600 \mathrm{~g} / \mathrm{mm}$ grating blazed at $240 \mathrm{~nm}$, $1800 \mathrm{~g} / \mathrm{mm}$ grating blazed at $500 \mathrm{~nm}$, and a $600 \mathrm{~g} / \mathrm{mm}$ grating blazed at $300 \mathrm{~nm}$. Detection system consisted of an Apogee spectral imaging camera with a Hamamatsu CCD detector $(2048 \times 128$ pixels $)$. The detector was calibrated with Spectra Physics black body irradiance source in the irradiance geometry that included reflections from cavity walls.

Apart from the omnipresent Ar I and O I lines, the optical spectra in the spectral range between 300 and $900 \mathrm{~nm}$ have revealed an abundant number of metal impurity lines 
and a number of molecular bands. Tentative identifications were made for Fe I, Ti I, Cr I, W I, Ni I, Sc I, Sr I, Nb I, Zr I, V I, Pb I, Cu I and $\mathrm{Cu}$ II, as well as Ne I, Ca I, C I, and $\mathrm{H}_{\alpha}$ lines. Metallic lines are suspected to be emitted from the evaporated metallic (possibly stainless steel) particulates. Neon is usually found as the impurity in Ar gas cylinder, and $\mathrm{C} \mathrm{I}$ and $\mathrm{H}_{\alpha}$ lines were the artifacts of the desorbed organic contaminants.

A number of molecular bands have offered the possibility for determining the extent of plasma based contaminant removal rates. For instance, the simultaneous presence of $\mathrm{OH}\left[\mathrm{A}^{2} \Sigma^{+} \rightarrow \mathrm{X}^{2} \Pi(0-0)\right]$ band at $306.4 \mathrm{~nm}$ and two vibrational bands, $\left[\mathrm{C}^{3} \Pi_{\mathrm{u}} \rightarrow \mathrm{B}^{3} \Pi_{\mathrm{g}}(1-0)\right]$ at $315.93 \mathrm{~nm}$ and $\left[\mathrm{C}^{3} \Pi_{\mathrm{u}}\right.$ $\left.\rightarrow \mathrm{B}^{3} \Pi_{\mathrm{g}}(2-1)\right]$ at $313.6 \mathrm{~nm}$, from $\mathrm{N}_{2}$ Second Positive System recorded in a common CCD frame (see Fig. 4) offers the possibility to determine the rate of removal of organic contaminants from the cavity walls since $\mathrm{N}_{2}$ concentration was stationary during spectral observation. Using the spectral data, we can identify the integral intensity ratio (IIR) of the $\mathrm{OH}$ and $\mathrm{N}_{2}$ signature spectra as

$$
\operatorname{IIR}\left\{[\mathrm{OH}] /\left[N_{2}\right]\right\}=\frac{\int_{\lambda=306 \mathrm{~nm}}^{\lambda=308 \mathrm{~nm}} \operatorname{Id} \lambda}{\int_{\lambda=314 \mathrm{~nm}}^{\lambda=316 \mathrm{~nm}} \operatorname{Id} \lambda} .
$$

This measure of the surface decontamination effect recorded on the time scale of about 20 min has provided the characteristic time for the effective disappearance of $\mathrm{OH}$ radical band from the plasma spectrum (see Fig. 5), which was determined to be about $16.7 \mathrm{~min}$.

Argon line intensities offer the possibility to determine effectively the plasma parameters. For instance, following the work of Boffard et al., ${ }^{21}$ it is possible to determine the electron temperature based on the ratio of intensities of the two Argon lines shown in Fig. 6. The ratio of the intensities of two lines, $\left\{5 \mathrm{p}^{2}[5 / 2] \rightarrow 4 \mathrm{~s}^{2}[3 / 2]^{\circ}\right\}$ at $420.07 \mathrm{~nm}$ and $\left\{5 \mathrm{p}^{2}[1 / 2] \rightarrow 4 \mathrm{~s}^{2}[3 / 2]^{\circ}\right\}$ at $419.83 \mathrm{~nm}$, depends on their

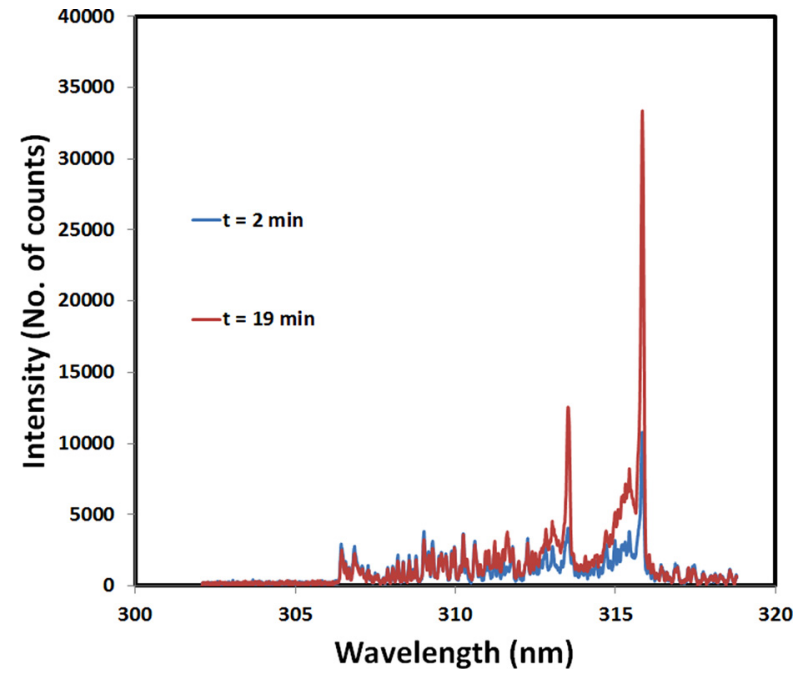

FIG. 4. Decaying presence of hydroxyl radical identified by the $\left[\mathrm{A}^{2} \Sigma^{+} \rightarrow\right.$ $\left.\mathrm{X}^{2} \Pi(0-0)\right]$ band at $306.4 \mathrm{~nm}$ signifies removal of hydrates or hydrocarbon films from cavity walls.

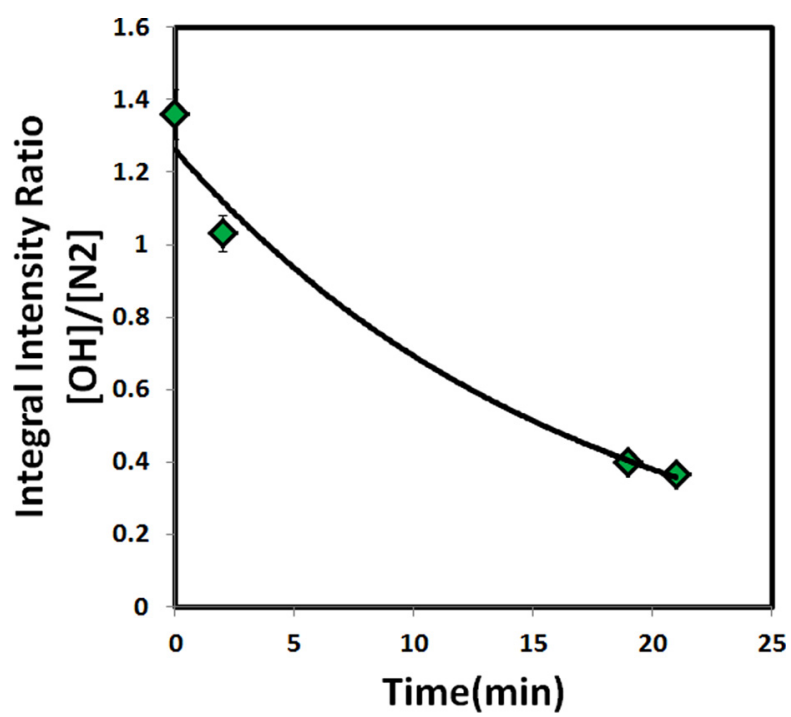

FIG. 5. Integral intensity ratio defined by Eq. (15) indicates the rate of removal of hydroxyl radical from the cavity walls.

excitation rates and the ratio of the metastable to groundstate population. Assuming a direct relationship between the metastable and ground-state population, one can determine electron temperature as the single free parameter from the experimental ratio of the intensities of the two lines. Thus determined the electron temperature of the plasma was about $3 \mathrm{eV}$. We note here that we were able to reproduce the average electron density using exclusively optical emission spectroscopy methods. First, gas temperature was obtained as the rotational temperature of the (0-2) spectral band of Nitrogen Second Positive System. This value was $600 \mathrm{~K}$. Second, the intensity ratio of two neighboring spectral lines of neutral and singly ionized argon was used together with the modified Saha equation ${ }^{22}$ to extract average electron density using the spectroscopically derived electron temperature of $3 \mathrm{eV}$. Thus applied Saha equation gave an estimated average electron density as $3.6 \times 10^{10} \mathrm{~cm}^{-3}$ in fair agreement with the value obtained from microwave measurements, which is given in Sec. IV B to be $2.8 \times 10^{10} \mathrm{~cm}^{-3}$.

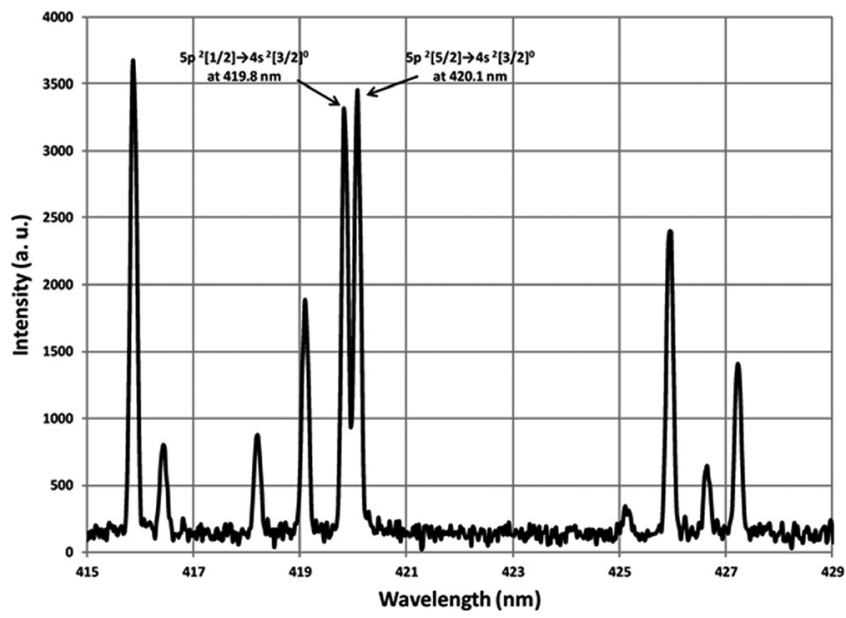

FIG. 6. Ar I spectral lines. Arrows indicate transitions used in the calculation. 
Compared to other discharge plasma, resonant microwave plasma has a relatively high electron temperature, which defines a high plasma potential and a robust voltage drop in the resonant cavity wall sheath. Consequently, the reactive ions are accelerated toward the wall up to high energy and are certainly capable of breaking molecular bonds of organic contaminants at the surface, and heating and evaporating materials from the particulates. This effect we could observe indirectly through the emission spectrum of hydroxyl radical, which could serve as an indicator for organic decomposition (see Figs. 4 and 5). Evaporation of particulates is observed by the excitation of low energy levels of metal atoms.

\section{CONCLUSION}

Large-volume microwave resonant plasma in $\mathrm{Ar} / \mathrm{O}_{2}$ was generated in a multi-cell radio-frequency cavity. Resonance discharge was established at the characteristic frequency of the cavity that was $1.5 \mathrm{GHz}$. Presence of plasma in the cavity volume induced the frequency shift, which required the power source frequency to be adjusted to establish the new resonant condition in the cavity and to produce higher plasma density. Using this approach, we could have applied the resonant frequency plasma to cleaning of the cavity surface. The low-pressure microwave breakdown condition based on the free diffusion coefficient and the diffusion length for cylindrical geometry fits rather well the experimental breakdown field data for Argon. Electron density build-up forms a negative feedback to the microwave electric field, produces another resonant condition where the plasma frequency equals the power source frequency, and limits the plasma parameters.

Resonant discharge was characterized by RF power measurements and optical emission spectroscopy. Electron density of the $\mathrm{Ar} / \mathrm{O}_{2}$ (90/10) plasma was about $2.8 \times 10^{10} \mathrm{~cm}^{-3}$, which was evaluated from the critical minimum of the transmitted power. The electron temperature, determined from the ratio of two neutral Argon line intensities at 419.8 and $420.1 \mathrm{~nm}$, was about $3 \mathrm{eV}$. Optical emission spectroscopy was employed to determine gas temperature to be $600 \mathrm{~K}$, and electron density to be $3.6 \times 10^{10} \mathrm{~cm}^{-3}$, which can be considered as a fair confirmation for attaining plasma frequency resonance. The discharge was generated and sustained at a single mode cavity resonant frequency and at a moderate power level. Volume power density and the relatively high electron temperature suggest that the conditions were met for abundant production of molecular and atomic oxygen reactive species. These were capable of sustaining a removal of volatile contaminant layer with the time constant for organics of about $20 \mathrm{~min}$.

\section{ACKNOWLEDGMENTS}

This work was supported by JSA/DOE Contract No. DE-AC05-060R23177. J. Upadhyay acknowledges the financial support from the Accelerator Division, Thomas Jefferson National Accelerator Facility under the same contract.

${ }^{1}$ H. F. Dylla, J. Nucl. Mater. 93/94, 61 (1980).

${ }^{2}$ W. Poschenrieder, G. Staudenmeier, and P. Staib, J. Nucl. Mater. 93/94, 322 (1980).

${ }^{3}$ G. Federici, R. A. Anderl, P. Andrew, J. N. Brooks, R. A. Causey, J. P. Coad, D. Cowgill, R. P. Doerner, A. A. Haasz, G. Janeschitz, W. Jacob, G. R. Longhurst, R. Nygren, A. Peacock, M. A. Pick, V. Philipps, J. Roth, C. H. Skinner, and W. R. Wampler, J. Nucl. Mater. 266-269, 14 (1999).

${ }^{4}$ I. Arkhipov, A. Gorodetsky, R. Zalavudtinov, V. Bukhovets, A. Zakharov, E. Mukhin, A. Razdobarin, V. Semenov, K. Vukolov, T. Mukhammedzyanov, Yu. Gott, A. Taranchenko, and S. Zvonkov, J. Nucl. Mater. 415, S1210 (2011).

${ }^{5}$ G. J. M. Hagelaar, D. Kogut, D. Douai, and R. A. Pitts, in 66th Annual Gaseous Electronics Conference, Princeton, NJ (Bull. Am. Phys. Soc., 2013), Vol. 58, p. 22.

${ }^{6}$ D. Mihailova, G. Haghelaar, P. Belenguer, C. Laurent, J. Lo, B. Caillier, L. Therese, and P. Guillot, in 66th Annual Gaseous Electronics Conference, Princeton, NJ (Bull. Am. Phys. Soc., 2013), Vol. 58, p. 78.

${ }^{7}$ H. F. Dylla, J. Vac. Sci. Technol. A 6, 1276 (1988).

${ }^{8}$ S.-H. Kim, M. Crofford, M. Doleans, J. Sunders, and J. Mammosser, in Proc. Part. Accel. Conf., New York, NY (2011), p. 2124.

${ }^{9}$ C. S. Hopper and J. R. Delayen, Phys. Rev. ST Accel. Beams 16, 102001 (2013).

${ }^{10} \mathrm{~S}$. C. Brown, Introduction to Electrical Discharges in Gases (Willey, New York, 1966).

${ }^{11}$ Yu. P. Raizer, Gas Discharge Physics (Springer-Verlag, Berlin, 1991).

${ }^{12}$ L. Celona, G. Ciavola, S. Gammino, N. Gambino, F. Maimone, D. Mascali, and R. Miracolia, TUCO-B03, Proc. ECRIS 08, Chicago, 2008.

${ }^{13}$ L. Tonks, Phys. Rev. 37, 1458 (1931).

${ }^{14}$ P. Leprince, G. Matthieussent, and W. P. Allis, J. Appl. Phys. 42, 412 (1971).

${ }^{15}$ L. Celona, S. Gammino, F. Maimone, D. Mascali, N. Gambino, R. Miracoli, and G. Ciavola, Eur. Phys. J. D 61, 107 (2011).

${ }^{16}$ M. Rašković, S. Popović, J. Upadhyay, L. Vušković, L. Phillips, and A.M. Valente-Feliciano, J. Vac. Sci. Technol. A 27(2), 301 (2009).

${ }^{17}$ M. Rašković, S. Popović, J. Upadhyay, L. Vušković, A.-M. ValenteFeliciano, and L. Phillips, Phys. Rev. ST Accel. Beams 13, 112001 (2010).

${ }^{18}$ J. Mammosser, S. Ahmed, K. Macha, J. Upadyay, M. Nikolić, S. Popović, and L. Vušković, in Proc. IPAC2012, New Orleans, LA (2012), p. 3156.

${ }^{19}$ S. Gammino, G. Ciavola, L. G. Celona, D. Mascali, and F. Maimone, IEEE Trans. Plasma Sci. 36, 1552 (2008).

${ }^{20}$ B. Agdur and B. Enander, J. Appl. Phys. 33, 575 (1962).

${ }^{21}$ J. B. Boffard, R. O. Jung, C. C. Lin, L. E. Aneskavich, and A. E. Wendt, J. Phys. D: Appl. Phys. 45, 045201 (2012).

${ }^{22}$ C. B. Collins, Phys. Rev. 158, 94 (1967). 